This document is the accepted manuscript version of the following article: Hariharan, P. S., Mariyatra, M. B., Mothi, E. M., Neels, A., Rosair, G., \& Anthony, S. P. (2017). Polymorphism and benzene solvent controlled stimuli responsive reversible fluorescence switching in triphenylphosphoniumfluorenylide crystals. New Journal of Chemistry. http://doi.org/10.1039/C7NJ01136A

\title{
ARTICLE
}

\section{Polymorphism and benzene solvent controlled stimuli responsive reversible fluorescence switching in triphenylphosphoniumfluorenylide crystals}

Received 00th January 20xx, Accepted 00th January 20xx

DOI: $10.1039 / x 0 x x 00000 x$

www.rsc.org/

\section{P. S. Hariharan, ${ }^{\mathrm{a}}$ M. Baby Mariyatra, ${ }^{\mathrm{b}}$ E. M. Mothi, ${ }^{\mathrm{c} *}$ Antonia Neels, ${ }^{\mathrm{d}}$ Georgina Rosair and Savarimuthu Philip Anthony ${ }^{\mathrm{a} *}$}

\begin{abstract}
Triphenylphosphoniumfluorenylide (TPPFY), a fluorescent fluorene attached molecule, showed polymorphism and benzene solvent induced aggregation enhanced emission (AEE) in solid state. Crystallization from $\mathrm{CH}_{3} \mathrm{CN}$ produced nonfluorescent crystals of TPPFY (TPPFY-1) whereas intense yellow fluorescent crystals (plates and blocks) were obtained from benzene (TPPFY-2, $\lambda \max =538 \mathrm{~nm}, \Phi f=38 \%$ ). Structural analysis indicates that TPPFY-1 exhibit strong $\pi \ldots \pi$ interactions (3.371 - $3.399 \AA$ A) between fluorene units in the crystal lattice that quenched the solid state fluorescence. In contrast, inclusion of benzene molecule in TPPFY-2 prevents close packing of fluorophores and showed intense yellow fluorescence in the solid state. TPPFY-2 showed only weak $\mathrm{C}-\mathrm{H} . . . \pi$ interactions between fluorene and phenyl group of triphenylphosphoniumylide. Hirshfeld surface analysis further supported the differences in the intermolecular interactions and molecular packing between TPPFY-1 and TPPFy-2. Interestingly, heating/strong crushing irreversibly converts fluorescent TPPFY-2 crystals to non-fluorescent TPPFY-1 crystals. However, recrystallization of TPPFY-1 from benzene produced fluorescent TPPFY-2 crystals. Thus TPPFY displays reversible off-on fluorescence switching heating/crushing and recrystallization. PXRD studies confirmed the polymorphism as well as phase conversion of TPPFY-2 to TPPFY-1 by external stimuli. This study indicates the role of benzene molecule on controlling molecular packing and functional properties of TPPFY.
\end{abstract}

\section{Introduction}

Molecular packing, conformation and intermolecular interactions play a significant role in controlling or modulating opto-electronic properties of functional organic molecules in the solid state. ${ }^{1}$ Subtle change in the conformation and intermolecular interaction produced different molecular arrangement and drastic modulation of electrical and fluorescent response in the solid state. ${ }^{2}$ Polymorphism, a chemical substance exist in more than one form in the crystalline state, is an important phenomenon that provides a method for better understanding the role of molecular packing and resulting solid state properties of organic functional molecules. $^{3}$ For example, four different polymorphs of pentacene showed different values of charge carrier mobility. ${ }^{4}$ Other organic semiconductors such as dithiophene-

\footnotetext{
Department of Chemistry, School of Chemical \& Biotechnology, SASTRA University, Thanjavur-613401, Tamil Nadu, India. E-mail: philip@biotech.sastra.edu.

${ }^{b .}$ Department of Chemistry, St. Xavier's College, Palayamkottai-627002, India centre for Scientific and Applied Research, PSN College of Engineering and Technology, Melathediyoor, Tirunelveli-627152, India. E-mail: mothichem@gmail.com

d. Center for X-ray Analytics, Empa-Swiss Federal Laboratories for Materials Science and Technology, Überlandstrasse 129, 8600 Dübendorf, Switzerland e. Institute of Chemical Sciences, School of Engineering \& Physical Sciences, HeriotWatt University, Edinburgh EH14 4AS, United Kingdom.

f. Electronic Supplementary Information (ESI) available: [PXRD pattern, molecular conformation and geometric parameters around phosphorus in the crystal lattice of TPPFY-1 and TPPFY-2. See DOI: 10.1039/x0xx00000x
}

tetrathiafulvalene and 1,3-diazapentacene are also known to exhibit polymorph dependent electrical properties. ${ }^{5}$

Polymorphism in organic solid state fluorescent materials has received increasing attention in recent years because it provides a simple strategy to generate tunable and switchable fluorescence without doing tiresome iterative synthesis. ${ }^{6}$ However, only limited number of organic fluorescence molecules exhibited polymorphism in the solid state. ${ }^{7}$ The polymorphs of 2,2':6,2'-terpyridine exhibit reversible structural change and rare on-off fluorescence switching by controlled heating. ${ }^{8}$ The solid state fluorescence of 9-anthrylpyrazole has been tuned from blue $(445 \mathrm{~nm})$ to greenish blue $(515 \mathrm{~nm})$ by polymorphism that showed different intermolecular interactions and molecular packing in the crystal lattice. ${ }^{9}$ The alkyl chain conformational change in tetracene lead to red and yellow emitting polymorphs. ${ }^{10}$ The arrangement of the anthracene unit in 9-anthrylpyrazole derivatives produced tunable fluorescence. ${ }^{11}$ The subtle change of quinacridone orientation in N,N'-di(nbutyl)quinacridone polymorphs produced different solid state fluorescence. ${ }^{12}$ Thermal induced molecular packing in excited state intra molecular proton transfer (ESIPT) and conformationally flexible molecules produced reversible fluorescence switching and tuning. ${ }^{13}$ The more twisted conformation in 2-(2-Hydroxyphenyl)-4(3H)-quinazolinone, an ESIPT molecule, lead to blue shifted emission whereas red shifted fluorescence has been reported for molecular packing with a flat conformation. ${ }^{14}$ 
In this manuscript we report the synthesis of fluorene attached triphenylphosphoniumfluorenylide (TPPFY) and its polymorphism dependent fluorescence in the solid state. Crystallization from $\mathrm{CH}_{3} \mathrm{CN}$ produced non-fluorescent crystals of TPPFY (TPPFY-1) whereas crystallization from benzene gave intense yellow fluorescent crystals (TPPFY-2, $\lambda_{\max }=538 \mathrm{~nm}, \Phi_{f}$ $=38 \%$ ). Single crystal analysis of TPPFY-1 revealed strong $\pi \ldots \pi$ stacking interactions between the fluorene units that leads to complete quenching of solid state fluorescence. The inclusion of benzene molecules in the crystal lattice of TPPFY-2 altered the intermolecular interactions and displayed strong solid state fluorescence. The strongly fluorescent TPPFY-2 crystals became non-fluorescent either by applying external force such as heating or strong mechanical crushing. Interestingly, recrystallization from benzene produced fluorescent TPPFY-2 again. PXRD studies clearly demonstrated the conversion of TPPFY-2 to TPPFY-1 by external stimuli. Thus molecular packing of TPPFY has been modulated by inclusion of benzene that leads to polymorphism and stimuli induced reversible fluorescence switching.

\section{Experimental Section}

Chemicals: Triphenylphosphine and 9-bromofluorene were purchased from Sigma-Aldrich and used without further purification. Other chemicals and solvents were purchased from Merck India.

\section{Synthesis of TPPFY}

A slight modification of the literature procedure ${ }^{15}$ has been used as described below. Triphenylphosphine (1.07 g, 4.07 $\mathrm{mmol})$ was dissolved in nitrobenzene $(10 \mathrm{~mL})$. To this a solution of 9-bromofluorene (1.00 g, $4.07 \mathrm{mmol})$ in nitrobenzene $(15 \mathrm{~mL})$ was added slowly in drops and stirred at RT for $2 \mathrm{~h}$ to afford fluorenyl-9-triphenylphosphonium bromide $(1.98 \mathrm{~g}, 3.90 \mathrm{mmol}, 95 \%)$ as colorless crystalline precipitate. A hot ethanolic solution of this bromide salt was made alkaline using ammonium hydroxide $(8 \mathrm{~mL})$ and stirring was continued for 30 mins. After cooling, triphenylphosphoniumfluorenylide was obtained as light yellow crystals, which were filtered and recrystallized using acetonitrile. Yield $1.45 \mathrm{~g}$, (87\%). IR ( $\left.\mathrm{KBr}, \mathrm{cm}^{-1}\right) 3434,3054$, 2988, 1601, 1583, 1546, 1479, 1464, 1430, 1321, 1306, 1278, $1212,1186,1151,1096,1074,1021,888,749,723,693,621$, 599, 542, 512, 455, 422. ${ }^{1} \mathrm{H} \mathrm{NMR}\left(\mathrm{CDCl}_{3}, 400 \mathrm{MHz}\right): 6.33\left(\mathrm{~d},{ }^{2} \mathrm{~J}=\right.$ 8.1), $6.88\left(\mathrm{t},{ }^{2} \mathrm{~J}=7.2\right), 6.99\left(\mathrm{t},{ }^{2} \mathrm{~J}=7.2\right), 7.48-7.81(\mathrm{~m}), 8.16\left(\mathrm{~d},{ }^{2} \mathrm{~J}=\right.$ 7.5). ${ }^{13} \mathrm{C} \mathrm{NMR}\left(\mathrm{CDCl}_{3}, 100 \mathrm{MHz}\right): 53.1$ (d, $\left.{ }^{1} \mathrm{~J}_{\mathrm{P}-\mathrm{C}}=128.1\right), 116.3(\mathrm{~d}$, $\left.{ }^{2} \mathrm{~J}_{\mathrm{P}-\mathrm{C}}=37.7\right) 119.5(\mathrm{~s}), 122.9(\mathrm{~s}), 125.8\left(\mathrm{~d},{ }^{1} \mathrm{~J}_{\mathrm{P}-\mathrm{C}}=89.0\right), 129.2(\mathrm{~d}$, $\left.{ }^{3} \mathrm{~J}^{\mathrm{P}-\mathrm{C}}=12.0\right), 130.9\left(\mathrm{~d},{ }^{4} \mathrm{~J}_{P_{-}-}=13.5\right), 132.8(\mathrm{~s}), 134.3\left(\mathrm{~d},{ }^{2} \mathrm{~J}_{P_{-}-}=9.8\right)$, $141.87\left(\mathrm{~d},{ }^{3} \mathrm{~J}_{\mathrm{P}-\mathrm{C}}=15.09\right) .{ }^{31} \mathrm{P} \mathrm{NMR}\left(\mathrm{CDCl}_{3}, 162 \mathrm{MHz}\right): 3.6$ (s).

\section{Spectroscopy and structural characterization}

Absorption and fluorescence spectra were recorded using Perking Elmer Lambda 1050 and Jasco fluorescence spectrometer-FP-8200 instruments. Fluorescence quantum yield ( $\Phi$ f) of TPPFY-2 was measured using a Horiba JobinYvon model FL3-22 Fluorolog spectrofluorimeter with integrating sphere. The powder X-ray diffraction (PXRD) patterns were measured using a XRD- Bruker D8 Advance XRD with $\mathrm{Cu} \mathrm{K \alpha}$ radiation $(\lambda=1.54050 \AA$ ) operated in the $2 \theta$ range from 100 to 50o. A yellow rod like shaped crystal of TPPFY-1 was mounted on a Stoe Mark II-Imaging Plate Diffractometer System (Stoe\&Cie, 2015) equipped with a graphite-monochromator. Data collection was performed at $173 \mathrm{~K}$ using Mo-Ka radiation $(\lambda=0.71073 \AA ̊ \Omega)$. Diffraction data for yellow block shaped crystal of TPPFY- 2 was collected at $100 \mathrm{~K}$ on Bruker X8 Apex 2 equipped with Mo-K $\alpha$ radiation $(\lambda=0.71073 \AA$ ) from a sealed tube with a graphite monochromator, a kappa goniometer and Apex2 CCD detector. CCDC Nos. 1497262 (TPPFY-1) and1497263 (TPPFY-2) contains the supplementary crystallographic data for this paper.

\section{Results and Discussion}

TPPFY was synthesized as shown in Scheme 1. The reaction between triphenylphosphine and 9-bromofluorene in nitrobenzene at room temperature produced fluorenyl-9triphenylphosphonium bromide that was basified and stirred to yield TPPFY as light yellow powder. TPPFY was synthesized as shown in Scheme 1 . The reaction between triphenylphosphine and 9-bromofluorene in nitrobenzene at room temperature produced fluorenyl-9triphenylphosphonium bromide that was basified and stirred to yield TPPFY as light yellow powder. The presence of fluorenyl group attached to $\mathrm{P}^{+}$centre stabilizes TPPFY via resonance where the negative charge on ylidic carbon can be delocalised over the aromatic fluorenyl group. The peak at $1186 \mathrm{~cm}^{-1}$ in the FTIR spectrum of TPPFY is assigned to $\mathrm{V}(\mathrm{P}=\mathrm{C})$ (Fig S1). This value is in good agreement with the range (1180 $-1230 \mathrm{~cm}^{-1}$ ) reported for the phosphorus ylides of the type $\mathrm{Ph} 3 \mathrm{P}=\mathrm{C} 5 \mathrm{H} 4$ where the $\mathrm{P}=\mathrm{C}$ is stabilized by powerful resonance interactions. ${ }^{16}$ In stark contrast, the peak observed at $1108 \mathrm{~cm}$ 1 in the IR spectrum of $\mathrm{TPPFY}^{+} \mathrm{Br}^{-}$is assigned to $\mathrm{V}(\mathrm{P}-\mathrm{C})$ confirming the increased $\mathrm{P}-\mathrm{C}$ bond order in TPPFY. Similarly, the ${ }^{31} \mathrm{P}$ NMR spectrum of TPPFY shows a single peak at 3.6 $\mathrm{ppm}$ which is much downfield shifted than ${ }^{31} \mathrm{P}$ NMR signal for $\mathrm{TPPFY}^{+} \mathrm{Br}^{-}$(30.6 ppm) further confirming the resonance stabilized interaction between phosphorus and carbon centres in TPPFY. The ${ }^{31} \mathrm{P}$ decoupled ${ }^{1} \mathrm{H}$ NMR spectrum shows well resolved signals accounting for all the protons and indicating the symmetry present in the molecule. $\mathrm{H} 4 / 4^{\prime}$ protons are appeared as doublet at $6.33 \mathrm{ppm}$ and $\mathrm{H} 7 / \mathrm{7}^{\prime}$ signal is shifted downfield and observed at $8.16 \mathrm{ppm}$ (Fig. S2). Two sets of triplet signals centred at 6.88 and $6.99 \mathrm{ppm}$ account for $\mathrm{H} 5 / 5^{\prime}$ and $\mathrm{H6} / 6$ ' protons, respectively. The three phenyl groups attached to phosphorus atom are observed as separate sets of multiplets in the region $7.48-7.81 \mathrm{ppm}$. The most interesting feature in the ${ }^{13} \mathrm{C}$ NMR spectrum is the high up-field shift of ylidic carbon, C1 (53.1 ppm) which hints that the negative charge is perhaps predominantly located at C1. All other carbons of fluorenyl group are located in the region 116.07 $141.86 \mathrm{ppm}$. The phenyl group carbons show separate signals for $C_{\text {ipso }}(125.8 \mathrm{ppm}), \mathrm{C}_{\text {ortho }}(134.3 \mathrm{ppm}), \mathrm{C}_{\text {meta }}(129.2 \mathrm{ppm})$ and $C_{\text {para }}(132.8 \mathrm{ppm})$. Coupling with $P$ centre is observed for most of the ${ }^{13} \mathrm{C}$ NMR signals. Among the carbons attached directly to $P$ atom, the greatest coupling constant value is noted for ylidic 
carbon, $\mathrm{C} 1\left({ }^{1} \mathrm{~J}_{\mathrm{P}-\mathrm{C}}=128.1 \mathrm{~Hz}\right)$ followed by phenyl ipso carbons $\left({ }^{1} \mathrm{~J}_{\mathrm{P}-\mathrm{C}}=89.0 \mathrm{~Hz}\right)$.<smiles>Cc1ccc2c(c1)C(Br)c1c[c-]ccc1-2</smiles><smiles>OC(O)c1ccc(P(=C2c3ccccc3-c3ccccc32)(c2ccccc2)c2ccccc2)cc1</smiles>

Scheme 1. Synthesis of TPPFY.

The attachment of fluorescent fluorene along with twisted non-planar conformation of phenyl group around phosphorus of TPPFY was expected to exhibit solid state fluorescence. However, the as-synthesized yellow powder of TPPFY did not show any florescence in the solid state. Single crystals of TPPFY was attempted to grow from different solvents $\left(\mathrm{CH}_{3} \mathrm{CN}, \mathrm{CH}_{2} \mathrm{Cl}_{2}\right.$, $\mathrm{CHCl}_{3}$, ethyl acetate, THF, $\mathrm{CH}_{3} \mathrm{OH}, \mathrm{C}_{2} \mathrm{H}_{5} \mathrm{OH}$ and benzene) to explore the polymorphism and get an insight on the molecular conformation and packing in the solid state. However, TPPFY single crystals were obtained only from $\mathrm{CH}_{3} \mathrm{CN}$ and benzene solvent by slow evaporation. The crystals grown from $\mathrm{CH}_{3} \mathrm{CN}$ are denoted as TPPFY-1 and crystals obtained from benzene are denoted as TPPFY-2. Similar to as-synthesized powder, TPPFY-1 did not show any solid state fluorescence. Interestingly, TPPFY crystals grown from benzene showed strong yellow fluorescence in the solid state (Fig. 1a inset). It is noted that TPPFY in solution state did not exhibit fluorescence in any solvents including benzene. This indicates the AEE phenomena of TPPFY-2 in the solid state. It was observed that only the crystals of TPPFY grown from benzene exhibited strong yellow fluorescence whereas powdered materials obtained by quick precipitation even from benzene did not show fluorescence. This suggests that molecular arrangement and conformation of TPPFY in the crystals play a significant role to produce solid state fluorescence.

Single crystal X-ray structural analysis was performed to get an insight on the molecular conformation and packing of TPPFY-1 and TPPFY-2. TPPFY-1 crystallized in the $\mathrm{P} 2{ }_{1} / \mathrm{c}$ space group whereas TPPFY-2 crystallized in $\mathrm{C} 2 / \mathrm{c}$ space group and confirmed the polymorphism (Table 1). Phosphorous in both crystals showed tetrahedral geometry with nearly similar bond distance and bond angle (Fig. S3, Table S1). However, twisting of phenyl and fluorene groups around phosphorous differed both in TPPFY-1 and TPPFY-2 (Fig. 1a). The molecular packing of TPPFY-1 showed $\pi \ldots \pi$ stacking interactions between fluorene (3.371-3.399 Å) that lead to the formation of face to face orientation of fluorene units and produced dimers $(\mathrm{H}-$ aggregate) in the crystal lattice with exactly opposite orientation of molecules (Fig. 1b). The weak $\mathrm{C}-\mathrm{H} \ldots \pi$ interactions further inter-connects the dimers in the crystal lattice along $a c$-plane. The formation of $\mathrm{H}$-aggregates with face to face orientation of fluorene in the crystal lattice makes it non-fluorescent in the solid state. The formation of a dimer splits excitation level into two levels: one lower and another higher than that of the monomer (Scheme S1). ${ }^{17}$ If the transition dipoles of the monomers are oppositely arranged, the electronic transition to and from the lower level is forbidden. However, the transitions to and from the higher level is optically allowed if coupling of dipoles results in a nonzero transition moment. Hence $\mathrm{H}$-aggregated solids showed blue shifted fluorescence often with lower efficiency since the exciton energy was consumed by vibration relaxation from upper level to low level, from which the transition to the ground state is forbidden. Hence TPPFY-1 that exhibited $\mathrm{H}$ aggregates with opposite dipole orientation is non-fluorescent in the solid state.

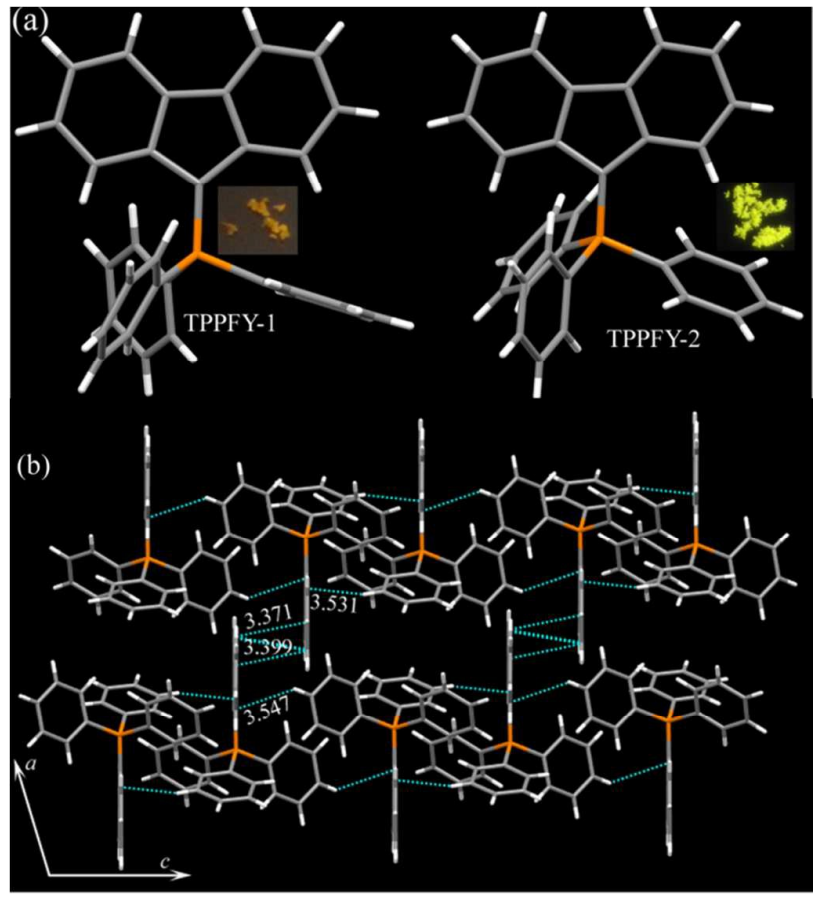

Fig. 1. (a) Molecular conformation of TPPFY in TPPFY-1 and TPPFY-2 and (b) intramolecular $\mathrm{H}$-bonding and $\pi \ldots \pi$ interactions in the crystals lattice of TPPFY-1. C (grey), $\mathrm{P}$ (orange), $\mathrm{H}$ (white); $\mathrm{H}$-bonds and $\pi \ldots \pi$ interactions (broken line). $\mathrm{H}$-bond $\pi \ldots \pi$ distances are marked in $\AA$.

The structural analysis of TPPFY-2 showed the inclusion of benzene molecules in the crystal lattice (Fig. 2a, S4). In contrast to TPPFY-1, TPPFY-2 did not show any $\pi \ldots \pi$ interactions and face to face orientation between fluorene units (Fig. 2). The fluorene unit formed only weak C-H... $\pi$ intermolecular interactions between phenyl hydrogen and fluorene (Fig. 2b). The two benzene molecules included in the crystal lattice of TPPFY-2 were adopted different orientations (Fig. 2a). Furthermore benzene molecules were stabilized via 
weak $\mathrm{C}-\mathrm{H} . . . \pi$ intermolecular interaction between of phenyl group and benzene solvent in the crystal lattice (Fig. 2c). Thus the benzene molecules play significant role in modulating intermolecular interactions and preventing face to face fluorene dimer formation in the crystal lattice of TPPFY-2. The prevention of face to face orientation of fluorene units resulted in strong fluorescence in the solid state. Differential scanning calorimetry (DSC) studies showed clear phase transition for TPPFY-2 at $88{ }^{\circ} \mathrm{C}$ whereas TPPFY-1 did not show any phase transition (Fig. S5).

In order to gain a deeper understanding and to quantify intermolecular interactions in the two polymorphs, Hishfeld surface analysis ${ }^{18}$ and associated 2D finger print plots $^{19}$ were generated using Crystal Explorer 3.1. ${ }^{20}$ The deep red spots in the $d_{\text {norm }}$ surfaces are indicative of hydrogen bonding contacts (Fig. 3a). In TPPFY-1, apart from the red spots over the phenyl rings, there is a prominent deep red region located over the centre of the fluorene moiety, whereas in TPPFY-2 there are two relatively smaller spots located on one side of the fluorene group (Fig. 3a) which clearly illustrates that the nature of intermolecular interactions are different in each of the

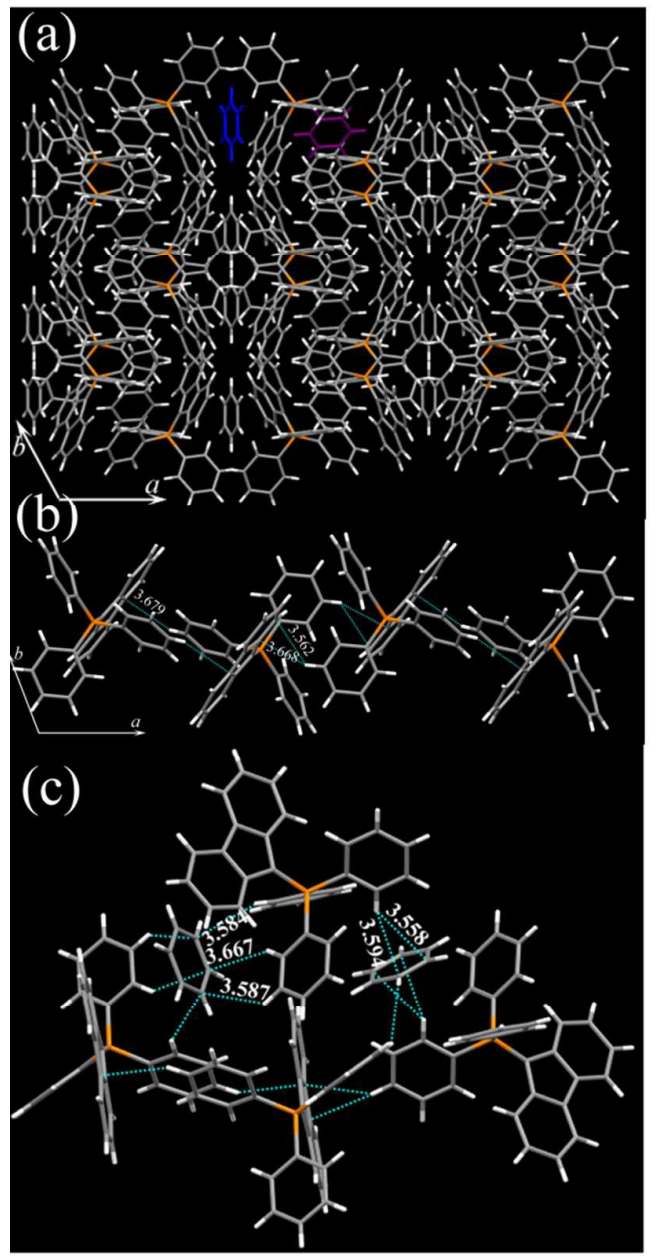

Fig. 2. Molecular packing (a) intermolecular interaction between TPPFY (b) and benzene intermolecular interaction with TPPFY in the crystals lattice of TPPFY-2. C (grey), P (orange), $\mathrm{H}$ (white); $\mathrm{H}$-bonds (broken line). $\mathrm{H}$-bond distances are marked in $\AA$.

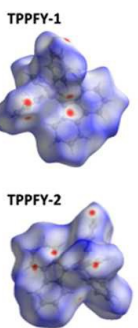

(a)
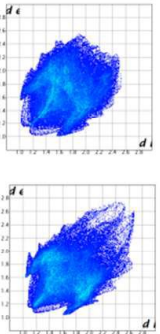

(b)
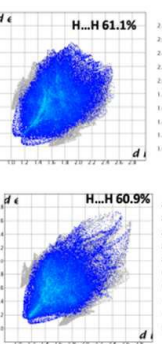

(c)
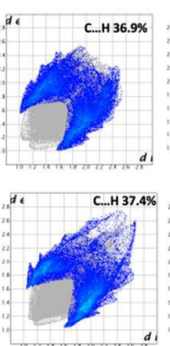

(d)

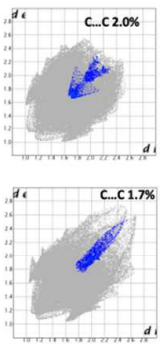

(e)
Fig. 3. Hirshfeld surfaces mapped with (a) $d_{\text {norm }}$ (b) finger print plots full, (c) resolved into $\mathrm{H} . . . \mathrm{H}$, (d) $\mathrm{C} . . . \mathrm{H}$ and (e) C...C (e) for TPPFY-1 and TPPFY-2 showing percentages of contact contributing to the total Hirshfeld surface area of the molecules.

Table 1. Crystallographic data of TPPFY-1 and TPPFY-2.

\begin{tabular}{|c|c|c|}
\hline Compound & $\begin{array}{c}\text { TPPFY-1 } \\
(1497262)\end{array}$ & $\begin{array}{c}\text { TPPFY-2 } \\
(1497263)\end{array}$ \\
\hline chemical formula & $\mathrm{C}_{31} \mathrm{H}_{23} \mathrm{P}$ & $\mathrm{C}_{31} \mathrm{H}_{23} \mathrm{P}, \mathrm{C}_{6} \mathrm{H}_{6}$ \\
\hline formula mass & 426.46 & 504.57 \\
\hline crystal system & monoclinic & monoclinic \\
\hline space group & P21/c (No. 14) & C2/c (No. 15) \\
\hline$a(\AA)$ & $13.252(3)$ & $27.996(2)$ \\
\hline$b(\AA ̊)$ & $16.668(2)$ & $11.8779(9)$ \\
\hline$c(\AA ̊)$ & $10.2556(18)$ & $17.3544(13)$ \\
\hline 8 (deg) & $103.378(14)$ & $113.260(3)$ \\
\hline $\begin{array}{c}\text { unit cell } \\
\text { volume }\left(\AA^{3}\right)\end{array}$ & $2203.8(7)$ & $5301.9(7)$ \\
\hline temp $(\mathrm{K})$ & 173 & 100 \\
\hline $\begin{array}{c}\text { no. of formula } \\
\text { units per unit cell, } \\
\text { Z }\end{array}$ & 4 & 8 \\
\hline radiation type & MoKa & MoKa \\
\hline abs coeff $\left(\mu / \mathrm{mm}^{-1}\right)$ & 0.142 & 0.129 \\
\hline no.reflns measured & 14169 & 51620 \\
\hline $\begin{array}{l}\text { no. independent } \\
\text { reflns }\end{array}$ & 3738 & 6565 \\
\hline$R_{\text {int }}$ & 0.099 & 0.058 \\
\hline $\begin{array}{c}\text { final } R_{1} \text { values }(I> \\
2 \sigma(I))\end{array}$ & 0.0454 & 0.0380 \\
\hline $\begin{array}{c}\text { final } R_{2}\left(F^{2}\right) \text { values }(I \\
>2 \sigma(I))\end{array}$ & 0.1205 & 0.0985 \\
\hline $\begin{array}{c}\text { final } R_{1} \text { values (all } \\
\text { data) }\end{array}$ & 0.0533 & 0.0598 \\
\hline $\begin{array}{c}\text { final } R_{2}\left(F^{2}\right) \text { values } \\
\text { (all data) }\end{array}$ & 0.1284 & 0.1189 \\
\hline GOF on $F^{2}$ & 1.08 & 1.11 \\
\hline
\end{tabular}

polymorphs. The H...H intermolecular contacts is the major contributor to the total Hirshfeld surface and comprises $61.1 \%$ in TPPFY-1 and $60.9 \%$ in TPPFY-2 (Fig. 3b). The characteristic spikes observed at the bottom left of finger print plot are associated with the $\mathrm{H}$...H contacts with the shortest of them occurring around $d_{\mathrm{e}}+d_{\mathrm{i}} \sim 2.2 \AA$ for both polymorphs (Fig. 3c). A clear splitting feature observed on the lower $d_{\mathrm{e}}$ and $d_{\mathrm{i}}$ distances of $\mathrm{H} . . . \mathrm{H}$ finger prints is indicative of the fact that a 
substantial number of shortest $\mathrm{H}$... $\mathrm{H}$ contacts are between three atoms and not between two direct atoms. ${ }^{20}$ The second dominant interaction observed in the finger print plot exhibited as a pair of well-defined peripheral "wings", is that of the H...C contacts comprising 36.9 and $37.4 \%$ in TPPFY- 1 and TPPFY-2, respectively to the total Hirshfeld surface (Fig. 3d). These wing-like features are recognized as characteristic of C$\mathrm{H} . . . \pi$ nature and the shortest of these H...C contacts are observed in the area around $d_{\mathrm{e}}+d_{\mathrm{i}} \sim 2.6 \AA$. The $\mathrm{C}$...C contacts comprises only $2.0 \%$ of total number of contacts in TPPFY-1 while the same contact for TPPFY-2 is slightly lower (1.7\%, Fig. 3e). The C...C contacts, which are related to $\pi \ldots \pi$ contacts are observed at the top right of the finger print plot as characteristic symmetric areas in the region $d_{\mathrm{e}}=d_{\mathrm{i}} \sim 1.7-2.2$ $\AA$ for TPPFY-1 which occurs as a broad feature whereas for TPPFY-2 it is observed at $d_{\mathrm{e}}=d_{\mathrm{i}} \sim 1.8-2.5 \AA$ as a narrow band. In addition, there is an interesting clear distinguishing feature observed between the finger print plots of the two polymorphs; only TPPFY- 2 shows a tail-like area at the top right of the plot. This tailing exhibited by a significant number of points at large values of $d_{\mathrm{e}}$ and $d_{\mathrm{i}}$ corresponds to regions that are devoid of any close contacts to nuclei in adjacent molecules. This is similar to what is observed in the finger print plots of benzene ${ }^{20}$ and other compounds containing phenyl groups. ${ }^{21}$ Thus, the difference in intermolecular interactions and packing is clearly reflected in the Hirshfeld surface and the corresponding finger print plots.

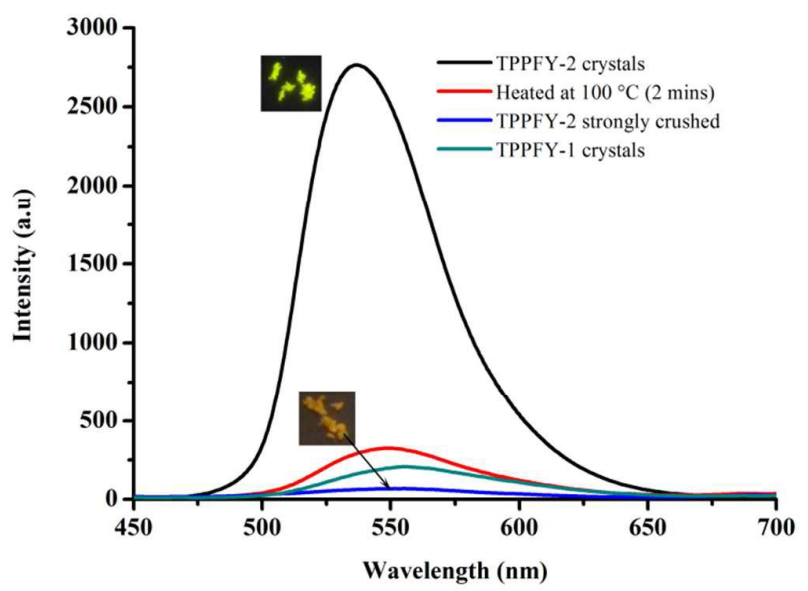

Fig. 4. Solid state fluorescence spectra of TPPFY-1 and TPPFY-2 under different stimuli.

The fluorescence spectra of TPPFY-2 showed strong solid state fluorescence at $\lambda_{\max }=538 \mathrm{~nm}\left(\Phi_{f}=38 \%\right.$, Fig. 4). Interestingly, mechanical crushing converted strongly fluorescent yellow TPPFY-2 crystals into completely nonfluorescent. Similarly, heating of TPPFY-2 crystals also converted to non-fluorescent form. We have also observed that TPPFY-2 becomes non-fluorescent after storing at room temperature for more than five days. This could be due to the loss of benzene molecules from the crystal lattice of TPPFY- 2 . However, unlike other organic fluorescent host systems ${ }^{22}$ that exhibits reversible fluorescence switching upon solvent exposure and removal, the solid state fluorescence of TPPFY-2 could not be recovered by exposing to benzene again. But recrystallization of non-fluorescent TPPFY powder from benzene converted back to yellow fluorescent crystals. Thus TPPFY becomes solid state fluorescence only when benzene molecules included in the crystals since that prevents $\pi \ldots \pi$ stacking interaction induced face to face orientation of fluorene in the crystal lattice. It is noted that most of mechanochromic fluorescent materials showed reversible fluorescence switching via phase change. ${ }^{23}$ To get an insight on the structural or phase change upon applying external stimuli (heating/mechanical crushing), we have performed PXRD for TPPFY-1 and TPPFY-2 (Fig. 5). The comparison of PXRD pattern of TPPFY-1 and TPPFY-2 with simulated pattern from respective single crystal data confirmed the phase purity of samples (Fig. S6,S7). Surprisingly, strong crushing or heating did not convert TPPFY-2 from crystalline to an amorphous phase but rather to the TPPFY-1 structure. However, exposure of benzene did not show reversible structural conversion. This could be due to the loss of weakly held benzene and higher stability of TPPFY-1 by strong $\pi \ldots \pi$ stacking interactions between fluorene units in the crystal lattice. We have also attempted to grow TPPFY crystals from toluene and xylene to study the effect of substitution in the benzene. Unfortunately, both toluene and xylene produced only non-fluorescent microcrystals of TPPFY.

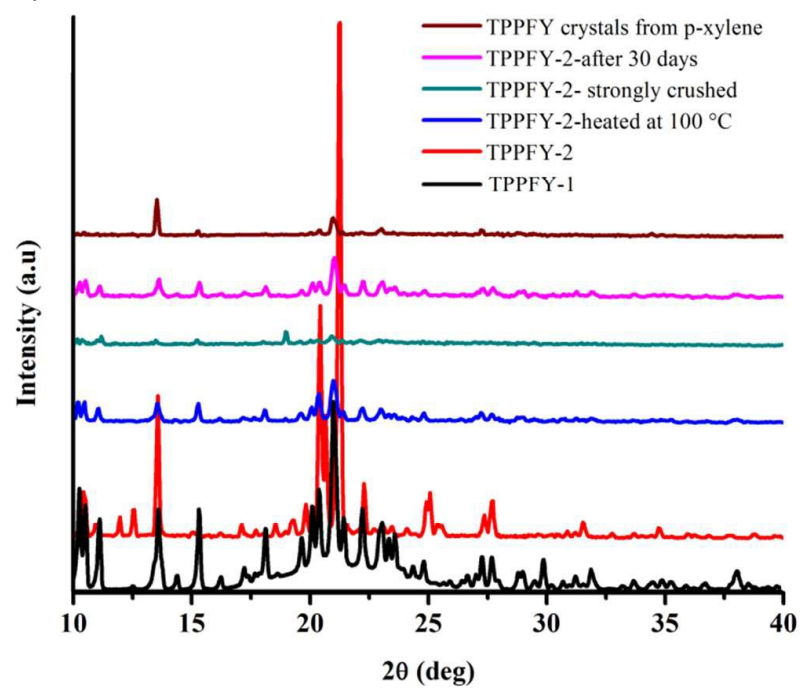

Fig. 5. PXRD pattern of TPPFY-1 and TPPFY-2.

\section{Conclusion}

In conclusion, we have synthesized fluorescent fluorene attached TPPFY and demonstrated polymorphism as well as benzene controlled stimuli responsive on-off fluorescence switching. TPPFY crystallized from $\mathrm{CH} 3 \mathrm{CN}$ revealed strong $\pi \ldots \pi$ stacking interactions between fluorene units in the crystal lattice that completely quenched the solid state fluorescence. In contrast, TPPFY-2 crystals grown from benzene showed strong yellow fluorescent $(\lambda \max =538 \mathrm{~nm}, \Phi f=38 \%$ ). TPPFY- 2 did not show any strong intermolecular interactions as well as 
$\pi \ldots \pi$ stacking interactions between fluorene units. The benzene molecules included in the crystal lattice completely modulated the molecular packing and intermolecular interactions. The weak intermolecular interactions between benzene and phenyl hydrogen stabilize benzene molecules in the crystals. The prevention of $\pi$... $\pi$ stacking between fluorene units lead to strong fluorescence in the solid state. TPPFY-1 was irreversibly converted to TPPFY-1 by heating or strong mechanical crushing. However, recrystallization from benzene produces fluorescent TPPFY-2 again. The difference in the intermolecular interactions and molecular packing was further confirmed by Hirshfeld surface analysis. Thus benzene solvent played significant role on the structural transformation as well as stimuli responsive reversible fluorescent properties of TPPFY in the solid state.

\section{Acknowledgement}

Financial supports from the Science and Engineering Research Board (SERB), New Delhi, India (SERB No. EMR/2015/00-1891, $\mathrm{SB} / \mathrm{FT} / \mathrm{CS}-182 / 2011$ ) is acknowledged with gratitude. EMM thanks SERB, India for young scientist grant (SB/FT/CS157/2012). The CRF facility of SASTRA University is also acknowledged for absorption spectroscopy. We thank Mr. P. Ramkumar, PSNCET for help in repeating the synthesis.

\section{Notes and references}

1 (a) R. Li, W. Hu, Y. Liu and D. Zhu, Acc. Chem. Res., 2010, 43, 529; (b) S.-J. Yoon and S. Y. Park, J. Mater. Chem., 2011, 21, 8338; (c) G. Zhang, J. Sun, P. Xue, Z. Zhang, P. Gong, J. Peng and R. Lu, J. Mater. Chem. C, 2015, 3, 2925; (d) S. Xue, X. Qiu, Q. Sun and W. Yang, J. Mater. Chem. C, 2016, 4, 1568; (e) W. Z. Yuan , Y. Tan , Y. Gong , P. Lu , Jacky W. Y. Lam , X. Y. Shen, C. Feng, Herman H-Y. Sung, Y. Lu , Ian D. Williams, J. Z. Sun , Y. Zhang and B. Z. Tang, Adv. Mater. 2013, 25, 2837.

2 (a) K. Wang, H. Zhang, S. Chen, G. Yang, J. Zhang, W. Tian, Z. Su and Y. Wang, Adv. Mater. 2014, 26, 6168; (b) A. G. Dikundwar, G. K. Dutta, T. N. Guru Row and S. Patil, Cryst. Growth Des. 2011, 11, 1615; (c) P. S. Hariharan, N. S. Venkataramanan, D. Moon and S. P. Anthony, J. Phys. Chem. C, 2015, 119, 9460; (d) P. S. Hariharan, D. Moon and S. P. Anthony, J. Mater. Chem. C, 2015, 3, 8381; (e) Y.-X. Li, H.-B. Zhou, J.-L. Miao, G.-X. Sun, G.-B. Li, Y. Nie, C.-L. Chen, Z. Chen and X.-T. Tao, CrystEngComm, 2012, 14, 8286.

3 (a) H. Y. Zhang, Z. L. Zhang, K. Q. Ye, J. Y. Zhang and Y. Wang, Adv. Mater. 2006, 18, 2369; (b) M. M. Bader, R. Custelcean and M. D. Ward, Chem. Mater. 2003, 15, 616; (c) G. Zhang, J. Lu, M. Sabat and C. L. Fraser, J. Am. Chem. Soc. 2010, 132, 2160; (d) X. Gu, J. Yao, G. Zhang, Y. Yan, C. Zhang and Q. Peng, Adv. Funct. Mater. 2012, 23, 4862; (e) R. Wei, P. Song and A. Tong, J. Phys. Chem. C, 2013, 117, 3467.

4 (a) A. Troisi and G. Orlandi, J. Phys. Chem. B 2005, 109, 1849; (b) C. C. Mattheus, A. B. Dros, J. Baas, G. T. Oostergetel, A. Meetsma, J. L. D. Boer and T. T. M. Palstra, Syn. Met. 2003, 138, 475; (c) A. Brillante, I. Bilotti, R. G. D. Valle, E. Venuti, M. Masino and A. Girlando, Adv. Mater. 2005, 17, 2549; (c) Q. Tang, D. Zhang, S. Wang, N. Ke, J. Xu, J. C. Yu and Q. Miao, Chem. Mater. 2009, 21, 1400.

5 (a) R. Pfattner, M. M. Torrent, I. Bilotti, A. Brillante, S. Milita, F. Liscio, F. Biscarini, T. Marszalek, J. Ulanski, A. Nosal, M. G. Lipman, M. Leufgen, G. Schmidt, L. W. Molenkamp, V.
Laukhin, J. Veciana and C. Rovira, Adv. Mater. 2010, 22, 4198; (b) A. Brillante, I. Bilotti, F. Biscarini, R. G. D. Valle and E. Venuti, Chem. Phys. 2006, 328, 125.

6 (a) S. P. Anthony, ChemPlusChem, 2012, 7, 518; (b) D. Frath, J. Massue, G. Ulrich and R. Ziessel, Angew. Chem. Int. Ed. 2014, 53, 2290; (c) N. S. S. Kumar, S. Varghese, N. P. Rath and S. Das, J. Phys. Chem. C 2008, 112, 8429; (d) A. Wakamiya, Y. Inukai and S. Yamaguchi, J. Am. Chem. Soc. 2006, 128, 15934; (e) A. Fernández-Mato, M. D. García, C. Peinador, J. M. Quintela, M. Sánchez-Andújar, B. Pato-Doldán, M. A. SeñarísRodríguez, D. Tordera and H. J. Bolink, Cryst. Growth Des. 2013, 13, 460; (f) Y. Zhang, Q. Song, K. Wang, W. Mao, F. Cao, J. Sun, L. Zhan, Y. Lv, Y. Ma, B. Zou and C. Zhang, J. Mater. Chem. C. 2015, 3, 3049; (g) M.-S. Yuan, D.-E. Wang, P. Xue, W. Wang, J.-C. Wang, Q. Tu, Z. Liu, Y. Liu, Y. Zhang and J. Wang, Chem. Mater. 2014, 26, 2467; (h) Z. He, L. Zhang, J. Mei, T. Zhang, J. W. Y. Lam, Z. Shuai, Y. Q. Dong and B. T. Tang, Chem. Mater. 2015, 27, 6601.

7 (a) R. Davis, N. P. Rath and S. Das, Chem. Commun. 2004, 74; (b) N. S. S. Kumar, S. Varghese, N. P. Rath and S. Das, J. Phys. Chem. C 2008, 112, 8429; (c) Y. Zhao, H. Gao, Y. Fan, T. Zhou, Z. Su, Y. Liu and Y. Wang, Adv. Mater. 2009, 21, 3165.

8 T. Mutai, H. Satou and K. Araki, Nat. Mater. 2005, 4, 685.

9 H. Y. Zhang, Z. L. Zhang, K. Q. Ye, J. Y. Zhang, and Y. Wang, Adv. Mater. 2006, 18, 2369.

10 C. Kitamura, T. Ohara, N. Kawatsuki, A. Yoneda, T. Kobayashi, H. Naito, T. Komatsuc and T. Kitamura, CrystEngComm, 2007, 9, 644.

11 Z. Zhang, Y. Zhang, D. Yao, H. Bi, I. Javed, Y. Fan, H. Zhang and Y. Wang, Cryst. Growth Des., 2009, 9, 5069.

12 Y. Fan, Y. Zhao, L. Ye, B. Li, G. Yang and Yue Wang, Cryst. Growth Des., 2009, 9, 1421.

13 (a) Y. Zhao, H. Gao, Y. Fan, T. Zhou, Z. Su, Y. Liu and Y. Wang Adv. Mater. 2009, 21, 3165; (b) T. Mutai, H. Tomoda, T. Ohkawa, Y. Yabe and K. Araki, Angew. Chem. Int. Ed. 2008, 47, 9522.

14 S. P. Anthony, Chem. Asian J. 2012, 7, 374.

15 A. Johnson, J. Org. Chem., 1959, 24, 282.

16 P. J. Taylor, Spectrochim. Acta, 1977, 34A, 115.

17 (a) M. Pope and C. E. Swenberg, Electronic Processes in Organic Crystals and Polymers, 2nd Ed., New York, 1999, 39; (b) M. Shimizu and T. Hiyama, Chem. Asian J. 2010, 5, 1516.

18 M. A. Spackman and D. Jayatilaka, CrystEngComm, 2009, 11, 19.

19 M. A. Spackman and J. J. McKinnon, CrystEngComm, 2002, 4, 378.

20 S. K. Wolff, D. J. Grimwood, J. J. McKinnon, M. J. Turner, D. Jayatilaka and M. A. Spackman, CrystalExplorer 3.1, University of Western Australia, 2012.

21 D. A. Safin, M. P. Mitoraj, K. Robeyns, Y. Filinchuk and C. M. L. V. Velde, Dalton Trans., 2015, 44, 16824.

22 (a) T. Han, X. Feng, J. Shi, B. Tong, Y. Dong, Jacky W. Y. Lam, Y. Dong and B. Z. Tang, J. Mater. Chem. C, 2013, 1, 7534; (b) S. P. Anthony, S. Varughese and S. M. Draper, Chem. Commun., 2009, 7500; (c) Y. Imai, K. Murata, K. Kawaguchi and T. Sato, Org. Lett., 2007, 9, 3457; (d) S. P. Anthony, C. Delaney, S. Varughese, L. Wang and S. M. Draper, CrystEngComm, 2011, 13, 6706.

23 (a) Y. Lv, Y. Liu, D. Guo, X. Ye, G. Liu and X. Tao, Chem. Asian J. 2014, 10, 2885; (b) Y. Dong, J. Zhang, X. Tan, L. Wang, J. Chen, B. Li, L. Ye, B. Xu, B. Zou and W. Tian, J. Mater. Chem. C, 2013, 1,7554; (c) Y. Zhang, J. Sun, X. Lv, M. Ouyang, F. Cao, G. Pan, L. Pan, G. Fan, W. Yu, C. He, S. Zheng, F. Zhang, W. Wang and C. Zhang, CrystEngComm, 2013, 15, 8998; (d) S.-J. Yoon, J. W. Chung, J. Gierschner, K. S. Kim, M.-G. Choi, D. Kim and S. Y. Park, J. Am. Chem. Soc. 2010, 132, 13675. 


\section{Polymorphism and benzene solvent controlled stimuli responsive}

\section{reversible fluorescence switching in triphenylphosphoniumfluorenylide crystals}

Triphenylphosphoniumfluorenylide (TPPFY), a fluorescent fluorene attached molecule, showed polymorphism, benzene solvent induced aggregation enhanced emission (AEE) and external stimuli responsive on-off fluorescence switching.

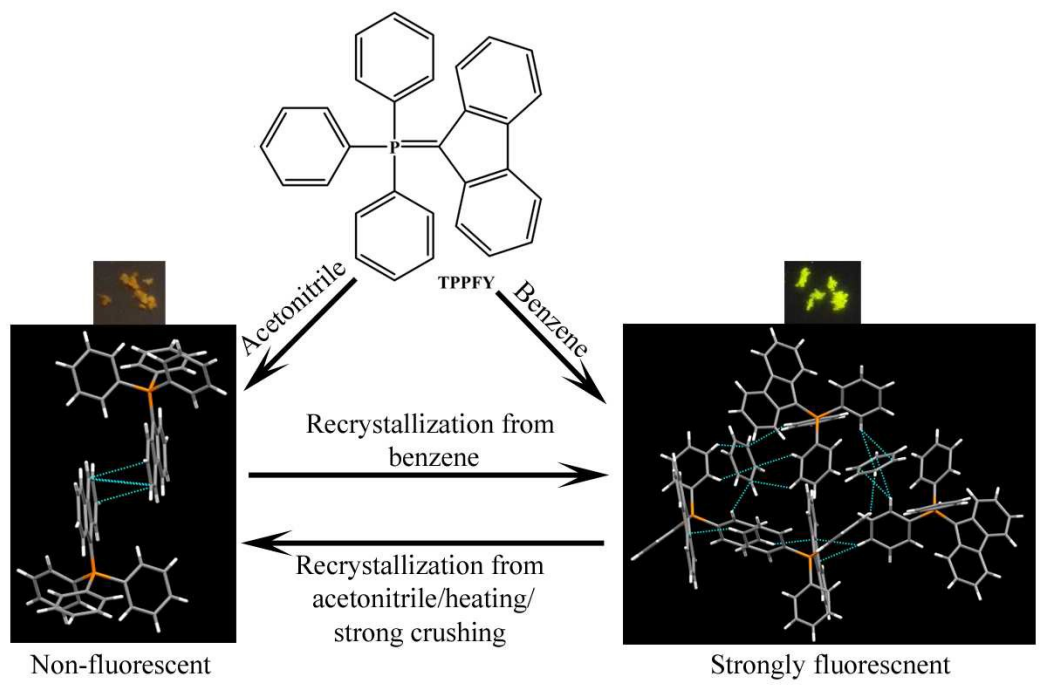

